\title{
EDITORIAL
}

\section{Farewell Missives}

\section{Stephen E. Braude}

https://doi.org/10.31275/20212369

Creative Commons License CC-BY-NC

The

his is a particularly rich issue of the JSE. And a hefty one. Its size is due primarily to two quite lengthy essays, one by Bryan Williams and one by Michael Sudduth. Of course, all of this issue's

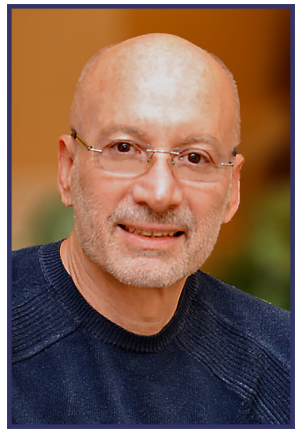
articles and reviews are worth reading; that's why we're publishing them. But these two huge essays merit a few extra comments.

Bryan Williams has given us something that I and various SSE members have hoped for over the years, a detailed review of a specific line of anomalistic research - the kind of article that would be useful to both veterans and newcomers to edge science. I've often tried to recruit such an opus from SSE colleagues at our conferences, hoping my considerable charm would dazzle them into accepting the opportunity. I've even been assured on several occasions that the solicited reviews would be forthcoming. But only Bryan, so far, has delivered the goods, a splendid essay surveying research on PK with random number generators. Not surprisingly, this review took Bryan a long time to write, and I want to thank him, not only for the result, but for his tenacity.

Michael Sudduth's essay is a forensic tour de force (as befits an admirer of the TV detective Columbo) - an unprecedently detailed critique of the James Leininger case of ostensible reincarnation. That case is both complicated and messy, and it illustrates a general problem with CORT investigations that I've dubbed the Problem of Investigative Intricacy.

All CORT cases are messy. Investigators must interview the subject, family members, and (when possible) crucial figures in the life of the 
previous personality. In fact, it typically requires considerable detective and interpretive work merely to identify the previous personality from the often vague or ambiguous behaviors and statements of the subject. And then, investigators must still obtain testimony from the remaining living persons (if any) who knew the apparent previous personality, simply to establish that the subject's apparent recollections about the previous personality are reliable. Moreover, the interview process can be muddied by the fallibility of memory, and by conscious or subconscious motives either to please (or frustrate) the interlocutor or simply to confirm a deep wish for the case to be a genuine instance of reincarnation. And of course, many cases also require the services of translators whose own biases, inadequacies, and needs might influence the direction or accuracy of the testimony obtained. So a great deal of work is required to obtain clear and reliable early-bird testimony (i.e., gathered before the apparent previous personality was identified), establish a strong link between that testimony (or the subject's behavior) and a previous personality, and to ensure the accuracy of the information obtained from interviews. In short, CORT investigations require a great deal of work simply to establish the reliable and relevant facts of the case.

However, other bodies of evidence don't require such a complex process either to identify the deceased or to establish a clear link between the living and the deceased. For example, in the case of the medium Mrs. Piper, we have many interactions between the medium and sitters who knew the deceased well. So when Mrs. Piper channeled a message having intimate relevance to the sitter (e.g., an affectionate and private nickname), we know immediately who the deceased seems to be. And arguably, transplant cases provide even clearer connections to the apparent deceased.

Sudduth's examination of the Leininger case is a breathtaking illustration of how difficult it is to properly evaluate evidence suggesting reincarnation. I imagine few of us have the fortitude or investigative skill for such a task. Indeed, I don't believe any ostensible survival case has ever been subjected to such a thorough examination. I should also note that Sudduth and Jim Tucker will have more to say about the Leininger case in the Spring issue. 
Before signing off permanently from my bully pulpit here at the JSE and passing the baton to James Houran, I would like to say a few words about Carlos Alvarado, whose recent and untimely death comes as a great loss to the JSE specifically and psi research generally. Alfonso Taboas has paid a splendid general tribute to Carlos in this issue, and I'd like to add that Carlos had a considerable influence on the JSE during my tenure as Editor-in-Chief. It was Carlos who pushed initially to establish an Historical Perspectives section to the Journal, and that innovation seems clearly to have been a success. In fact, many of the historical papers we've published are also our most frequently downloaded. Moreover, Carlos was an effective and pro-active Associate Editor as well as a frequent contributor to the Journal. He was also quick to inform me about new books deserving to be reviewed in our pages. Without question, he made my job easier, and I mourn the loss of a great colleague, good friend, and wonderful person.

Now that I'm preparing to enter that purgatory reserved for retiring Editors, I must also acknowledge the other members of my editorial team who've contributed so much to maintaining the scientific and scholarly integrity of the JSE. Because the JSE considers more areas of anomalistics than any one editor can hope to master, I've often had to rely on Associate Editors whose areas of specialization and competence differ from mine. Fortunately, my AEs have been remarkably supportive and helpful, and l'll no doubt miss our frequent exchanges. During my 13 years as Editor-in-Chief, my team has undergone inevitable changes in personnel, too many to list here. At any rate, I want to remind readers that our AEs are vital actors behind the scenes. I'd like, therefore, to acknowledge the following intrepid souls who've (at one time or another during my tenure) served as Associate Editors: Carlos Alvarado, Imants Barušs, Daryl Bem, Robert Bobrow, Etzel Cardeña, Courtney Brown, Jeremy Drake, Renaud Evrard, Hartmut Grote, Bernard Haisch, Michael Ibison, John Ives, Julia Mossbridge, Roger Nelson, Dean Radin, Mark Rodeghier, Daniel Sheehan, Paul Smith, James Spottiswoode, Michael Sudduth, and Harald Walach. 
And finally, I must once again express my appreciation for Managing Editor Kathleen Erickson, whose mastery of the mechanics of publishing is awe-inspiring, and who (as I've noted previously) manages the seemingly impossible task of preserving the illusion of my editorial competence. Kathleen has been a delight to work with, and I know that my Associate Editors and contributors to the Journal agree wholeheartedly. 